\title{
A Comprehensive Functional Analysis in Patients after Atrial Switch Surgery
}

\author{
Andreas Hornung ${ }^{1, *}$ Daniela Vollmer ${ }^{1, *}$ \\ Michael Hofbeck ${ }^{1}$ Ludger Sieverding ${ }^{1}$ \\ 1 Department Pediatric Cardiology, Pulmonology and Intensive Care, \\ University Children's Hospital Tuebingen, Tuebingen, Germany \\ 2 Department Pediatric Cardiology, University Children's Hospital \\ Ulm, Ulm, Germany \\ ${ }^{3}$ Department Pediatric Cardiology and Congenital Heart Disease, \\ German Heart Center, Munich, Germany
}

Thorac Cardiovasc Surg 2021;63:e32-e40.

Address for correspondence Andreas Hornung, MD, Hoppe-SeylerStr. 1, 72076 Tuebingen, Germany (e-mail: andreas.hornung@med.uni-tuebingen.de).

\begin{abstract}
Keyword

- congenital heart disease

- CHD

- heart failure

- imaging (all modalities)

- Conductance

Background Long-term course after atrial switch operation is determined by increasing right ventricular (RV) insufficiency. The aim of our study was to investigate subtle functional parameters by invasive measurements with conductance technique and noninvasive examinations with cardiac magnetic resonance imaging (CMR).

Methods We used invasive (pressure-volume loops under baseline conditions and dobutamine) and noninvasive techniques (CMR with feature tracking [FT] method) to evaluate RV function. All patients had cardiopulmonary exercise testing (CPET).

Results From 2011 to 2013, 16 patients aged $28.2 \pm 7.3$ (22-50) years after atrial switch surgery (87.5\% Senning and $12.5 \%$ Mustard) were enrolled in this prospective study. All patients were in New York Heart Association (NYHA) class I to II and presented mean peak oxygen consumption of $30.1 \pm 5.7(22.7-45.5) \mathrm{mL} / \mathrm{kg} / \mathrm{min}$. CMR-derived end-diastolic volume was $110 \pm 22(78-156) \mathrm{mL} / \mathrm{m}^{2}$ and RV ejection fraction $41 \pm 8 \%(25-52 \%)$. CMRFT revealed lower global systolic longitudinal, radial, and circumferential strain for the systemic RV compared with the subpulmonary left ventricle. End-systolic elastance (Ees) was overall reduced (compared with data from the literature) and showed significant increase under dobutamine $(0.80 \pm 0.44$ to $1.89 \pm 0.72 \mathrm{~mm} \mathrm{Hg} / \mathrm{mL}, p \leq 0.001)$, whereas enddiastolic elastance (Eed) was not significantly influenced $(0.11 \pm 0.70$ to $0.13 \pm 0.15 \mathrm{~mm}$ $\mathrm{Hg} / \mathrm{mL}, p=0.454)$. We found no relevant relationship between load-independent conductance indices and strain or CPET parameters. Conductance analysis revealed significant mechanical dyssynchrony, higher during diastole (mean $30 \pm 4 \%$ baseline, $24 \pm 6 \%$ dobutamine) than during systole (mean $17 \pm 6 \%$ baseline, $19 \pm 7 \%$ dobutamine).

Conclusions Functional assessment of a deteriorating systemic RV remains demanding. Conductance indices as well as the CMR-derived strain parameters showed overall reduced values, but a significant relationship was not present (including CPET). Our conductance analysis revealed intraventricular and predominantly diastolic RV dyssynchrony.
\end{abstract}

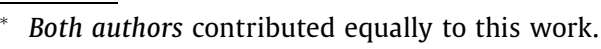

received

May 11,2020

accepted after revision

January 25, 2021
DOI https://doi.org/ $10.1055 / \mathrm{s}-0041-1726307$ ISSN 0171-6425.

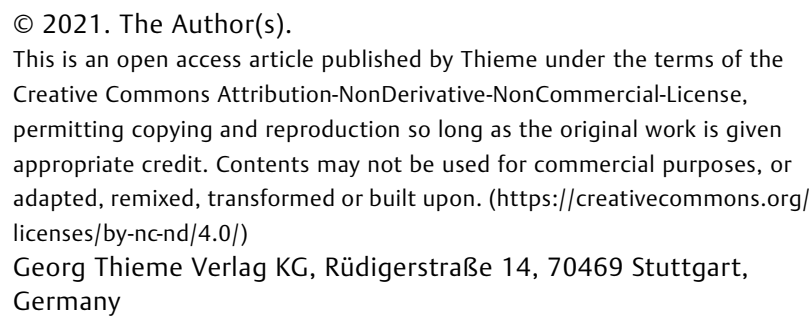




\section{Introduction}

Early detection of impairment of systemic right ventricular (RV) function is still demanding in patients after atrial switch procedure. Despite a detailed monitoring of the patients after atrial correction, there is no sensitive indicator that allows early detection of RV dysfunction at an early stage and marks the onset of progressive right heart failure. ${ }^{1}$ Sensitive indicators could be beneficial to develop effective therapy concepts in this patient group. The aim of our study was to investigate subtle functional parameters by invasive measurements with a pressure-volume conductance system (under baseline conditions and under dobutamine application) as well as by noninvasive examinations with cardiac magnetic resonance imaging (CMR). We also wanted to investigate whether invasive parameters correlate with noninvasive data. Besides, all patients had a cardiopulmonary exercise testing (CPET).

\section{Methods}

All patients with D-transposition of the great arteries (DTGA) after atrial switch and the indication for an invasive hemodynamic evaluation by routine cardiac catheterization were enrolled in this prospective, nonrandomized, monocentric study. Indications for cardiac catheterization were the need for hemodynamic assessment, suspicion of a baffle leak, or systemic and/or pulmonary venous tunnel obstruction (with the possibility of intervention). Patients with pacemakers were excluded due to changed hemodynamics. Written informed consent was given by all patients to a protocol approved by the ethics committee of the University Hospital Tuebingen. The CMR and conductance data were collected in accordance with our formerly published study protocol. ${ }^{2}$ For better comparability with literature, calibration of conductance was performed using thermodilution. The New York Heart Association (NYHA) functional class was recorded in all patients. The QRS duration was measured in lead II of a standard electrocardiogram (ECG). In each patient, the N-terminal pro-brain natriuretic peptide (NT-proBNP) level was measured at admission for cardiac catheterization (ethylenediaminetetraacetic acid [EDTA] blood was analyzed on the device ADVIA Centaur XP Immunoassay System, Siemens Healthineers, Erlangen, Germany).

\section{Cardiac Catheterization}

All cardiac catheterizations were performed under general anesthesia. After routine catheterization, the conductance catheter 71083-PL or 71083-PN (specifications: 12 electrodes, max. 7 segments, interelectrode distance $8 \mathrm{~mm}$, pigtail tip $1.5 \mathrm{~cm}$, CD Leycom, Zoetermeer, The Netherlands) was carefully positioned via femoral artery into the outflow tract and RV apex under fluoroscopic guidance. In addition, the quality of the segmental pressure-volume loops was checked before rest and stress measurements.

The analysis unit CFL-512 (CD Leycom) recorded real-time pressure-volume curves under a short, end-expiratory ventilation pause. The technical background and principles of the conductance technique have been previously described in existing publications. ${ }^{3}$ Blood conductivity was measured over up to seven segments from apex to base depending on the ventricular size. For volume calibration, a minimum of three thermodilution measurements were performed using the Picco catheter (Pulsion, Munich, Germany) during baseline recording with calculation of an average value. To obtain load-independent parameters of systemic RV function, preload reduction was achieved by inflation of a balloon catheter in the inferior caval vein (PTS Sizing Balloon, Fa Numed Inc.; size: $20-25 \mathrm{~mm}$ ). The pressure-volume loops were evaluated at rest and under a steady-state dobutamine infusion with a maximal dosage of $10 \mu \mathrm{g} / \mathrm{kg} / \mathrm{min}$. Dobutamine dosage was reduced in case of excessive increase in blood pressure or heart rate, also in cases of high-grade arrhythmia. Calibration and evaluation of the data was performed offline with dedicated analysis software INCA Conduct NT (Version 3.18.1 of CD Leycom).

Mechanical ventricular dyssynchrony in a volume segment exists if this segment is not involved in the overall volume change or even shows opposite volume changes. ${ }^{4}$ Segmental dyssynchrony was quantified as the deviation of a single segment versus total volume change (per time period in percent). Dyssynchrony of the whole ventricle was defined as mean value of all segmental dyssynchronies. Because of the varying number of evaluable segments in our patients (ventricle size and differences in contractility), the dyssynchrony parameters were converted into five segments (with weighted percentages). Systolic dyssynchrony was defined as period between end-diastolic pressure (Ped) and end-systolic pressure (Pes), and diastolic dyssynchrony between Pes and Ped (patient example under - Fig. 1).

\section{Cardiopulmonary Exercise Testing}

All patients underwent CPET on a treadmill with monitoring of expiratory gases according to the protocol of the German Society for Pediatric Cardiology (DGPK). ${ }^{5}$ The evaluations included the values for peak oxygen consumption $\left(\mathrm{pVO}_{2}\right)$, peak oxygen pulse (as a quotient of $\mathrm{pVO}_{2}$ and peak heart rate), heart rate reserve (as a difference of peak exercise heart rate and resting heart rate), and the respiratory exchange ratio. Target rates were defined as percentages of the ageand sex-specific 50th percentile of standard values specified by Dubowy et al. ${ }^{5}$

\section{Cardiac Magnetic Resonance Imaging Study}

With a 1.5-T MR system (Magnetom SonataVision and Espree, Siemens Healthcare, Erlangen, Germany), ECG-triggered 2D cine steady-state free precession (SSFP) sequences were acquired during breath hold to assess the RV function. An axial stack of the whole heart served as the basis for volumetric analysis (specifications: slice thickness of 5-6 $\mathrm{mm}, 20$ phases, heart rate-adapted repetition time [TR], echo time [TE] of 1.23 milliseconds, flip angle of 43 degrees, bandwidth of $1,184 \mathrm{~Hz} /$ pixel, acquisition matrix of $192 \times 127$, field of view [FOV] of $280-340 \mathrm{~mm}$ ). Postprocessing was done with Argus software (Siemens Healthineers) in which endocardial borders in the end-systolic and 
a)

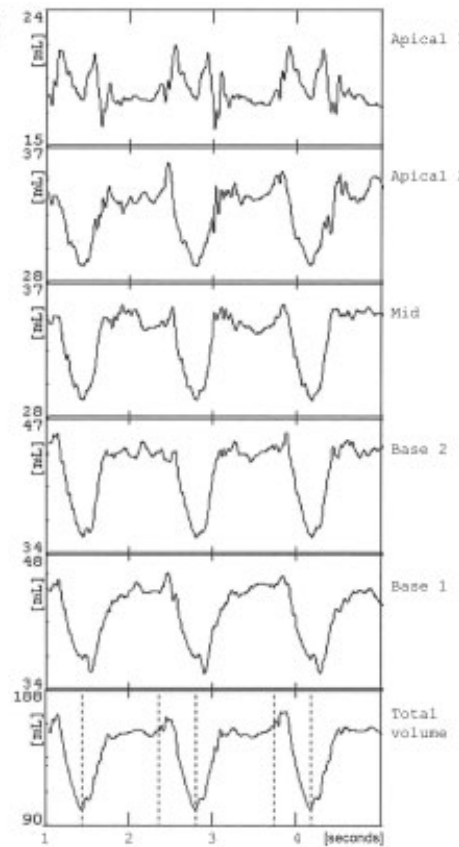

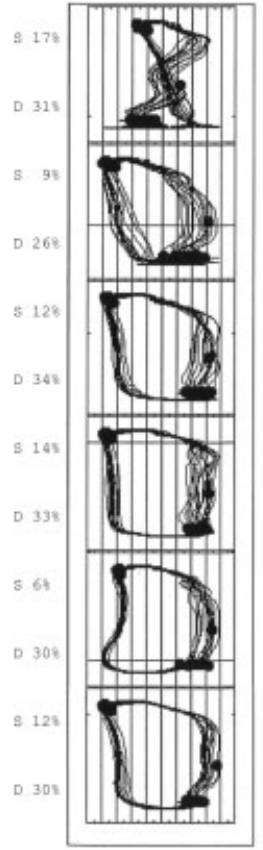

b)

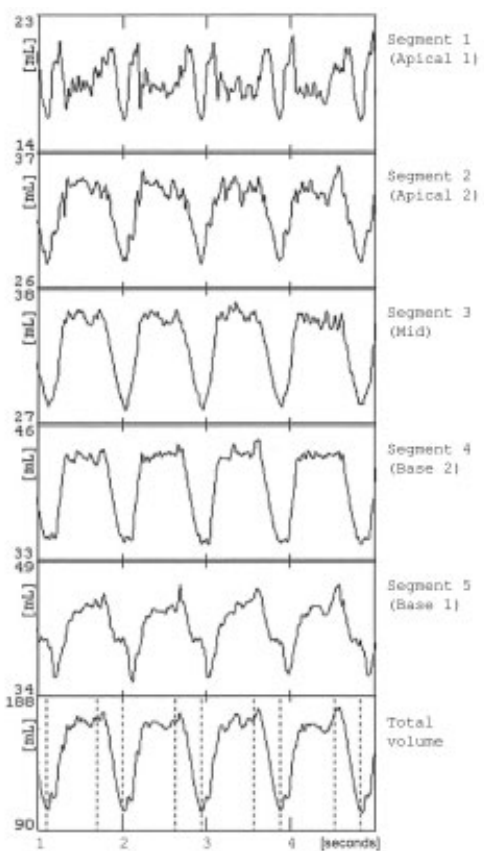

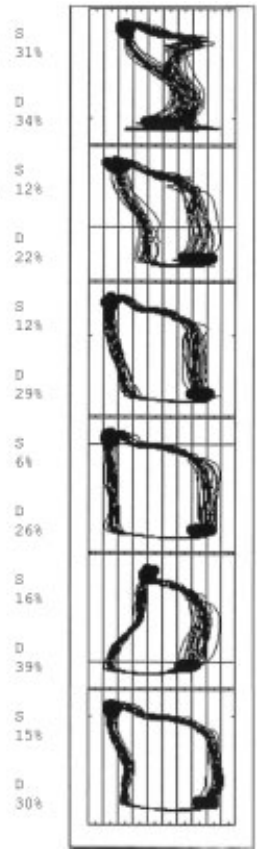

Fig. 1 Segmental volume-time data as well as corresponding pressure-volume loops and values of systolic (S) and diastolic (D) dyssynchrony (a) at rest and (b) during dobutamine infusion.

the end-diastolic phase were semiautomatically determined for all axial slices. Flow measurements in the pulmonary artery and in the ascending aorta were performed by using free breathing 2D phase-contrast sequences (slice thickness: 5-6 mm; 20 phases).

The cine sequences of the four-chamber view and the mid short axis were exported in DICOM format for postprocessional analysis with feature tracking (FT; Software 2D-CPAMR and ImageArena V4.6, TomTec, Unterschleissheim, Germany). Technical principles of FT and reference values for this specific patient group have been previously published. ${ }^{6,7}$ The values for maximal systolic global longitudinal strain (GLS), global radial strain (GRS), and global circumferential strain (GCS) were evaluated for the systemic right and the subpulmonary left ventricle. GLS and GRS were analyzed in the four-chamber view and GCS in the mid short axis. Endocardial borders were semiautomatically traced and visually assessed during the cardiac cycle. In case of marked deviation, the endocardial line was corrected manually.

\section{Statistical Analysis}

Descriptive statistics included calculation of mean value and standard deviation (mean \pm SD), partly supplemented by the data area (range). Changes between measurements at rest and under dobutamine infusion were expressed in percent increase (\%). The paired sign test was used to test for significant changes. Relationships between individual parameters were calculated using the Spearman correlation coefficient. Correlations were defined as statistically significant if the $p$-value was less than 0.05 . For statistical analysis, the software SPSS was used (Version 21, IBM, Chicago, Illinois, United States).

\section{Results}

\section{Patient and Clinical Characteristics}

A total of 18 patients fulfilled the inclusion criteria. In 16 cases (mean age: $28.2 \pm 7.3$ years; range: $22-50$ years), an evaluable series of pressure-volume loops at rest and under dobutamine infusion (with and without preload reduction) maneuver could be performed. The majority of patients (88\%) underwent Senning procedure, whereas only two patients had Mustard repair. The mean age at repair of our predominantly male patients (81\%) was $1.1 \pm 0.8$ years (0.6-3.42 years; - Table $\mathbf{1})$.

All patients were in NYHA class I to II ( - Table 1 ). Mean QRS duration was not relevantly prolonged $(103 \pm 20$ milliseconds; range: 70-158 milliseconds). Mean NT-proBNPlevel was not elevated $(152 \pm 110 \mathrm{ng} / \mathrm{L}$; range: $35-425 \mathrm{ng} / \mathrm{L})$ with a reference value $\leq 300 \mathrm{ng} / \mathrm{L}$. Only one patient had an elevated NT-proBNP level with $425 \mathrm{ng} / \mathrm{L}$. Five patients showed aortic insufficiency $I^{\circ}$ (4-6\% of stroke volume [SV]), five patients tricuspid insufficiency I ${ }^{\circ}(6-16 \%$ of SV), and two patients tricuspid insufficiency II ${ }^{\circ}(20-26 \%$ of SV). Two patients were under cardiac medication (12.5\%): one patient with NYHA class I under antihypertensive medication and another patient with NYHA class II (and elevated NT-proBNP level with $425 \mathrm{ng} / \mathrm{L}$ ) under heart failure medication (-Table $\mathbf{1})$.

\section{Cardiopulmonary Exercise Testing}

On average, the heart rate increased to $89 \%$ of the target rate. In all patients, a respiratory quotient greater than 1 was reached. The mean peak oxygen pulse $\left(\mathrm{O}_{2} / \mathrm{HR}\right)$ was $14.4 \pm 3.7$ $\mathrm{mL} /$ beat and showed a high spread $(8.3-23.1 \mathrm{~mL} /$ beat $)$; the mean peak oxygen consumption (peak $\mathrm{VO}_{2}$ ) was $30.1 \pm 5.7$ 
Table 1 Patient characteristics, clinical, and spiroergometric data

\begin{tabular}{|c|c|}
\hline Patients (n) & 16 \\
\hline Male & $13(81 \%)$ \\
\hline Female & $3(19 \%)$ \\
\hline $\operatorname{BSA}\left(\mathrm{m}^{2}\right)$ & $1.93 \pm 0.26(1.54-2.50)$ \\
\hline Age at study $(y)$ & $28.2 \pm 7.3(22-50)$ \\
\hline Age at repair (y) & $1.1 \pm 0.8(0.6-3.4)$ \\
\hline Senning & $14(88 \%)$ \\
\hline Mustard & $2(13 \%)$ \\
\hline Baffle leak & $3(19 \%)$ \\
\hline Baffle stenosis & $4(31 \%)$ \\
\hline $\mathrm{AlI}^{\circ}$ & $5(4-6 \%)$ \\
\hline $\begin{array}{l}\text { Tricuspid insufficiency } \\
\text { (TI) I (range: \%SV) }\end{array}$ & $5(6-16 \%)$ \\
\hline TI II (range: \%SV) & $2(20-26 \%)$ \\
\hline EPS (atrial dysrhythmia) & $2(13 \%)$ \\
\hline NYHA I & $9(56 \%)$ \\
\hline NYHA II & $7(43 \%)$ \\
\hline QRS duration (ms) & $103 \pm 20(70-158)$ \\
\hline NT-proBNP (ng/L) & $152 \pm 110(35-425)$ \\
\hline Cardiac medication & $2(13 \%)$ \\
\hline $\begin{array}{l}\text { Peak oxygen consumption } \\
\left.\text { (mL } \mathrm{O}_{2} / \mathrm{kg} / \mathrm{min}\right)\end{array}$ & $30.1 \pm 5.7(22.7-45.5)$ \\
\hline Peak oxygen pulse (mL/beat) & $14.4 \pm 3.7(8.3-23.1)$ \\
\hline $\mathrm{VE} / \mathrm{VCO}_{2}$ slope & $24.2 \pm 4.1(15.9-30.4)$ \\
\hline Peak SBP (mm Hg) & $162 \pm 20(109-195)$ \\
\hline Heart rate reserve (\%) & $89 \pm 9(68-101)$ \\
\hline RER & $1.13 \pm 0.08(1.01-1.30)$ \\
\hline
\end{tabular}

Abbreviations: Al, aortic insufficiency; BSA, body surface area; EPS, electrophysiological study; NYHA, New York Heart Association; NT-proBNP, $\mathrm{N}$-terminal pro-brain natriuretic peptide; RER, respiratory exchange ratio; SBP, systolic blood pressure; SV, stroke volume; TI, tricuspid insufficiency; $\mathrm{VE} / \mathrm{VCO}_{2}$, minute ventilation/carbon dioxide production.

Note: Data are means \pm standard deviation (percantage or range).

$\mathrm{mL} / \mathrm{kg} / \mathrm{min}$ (-Table $\mathbf{1}$ ). Oxygen pulse was negatively correlated to arterial elastance (Ea; resting conditions: $r=-0.511$ with $p=0.043$; under exercise: $r=-0.627$, with $p=0.009$ ). No statistically relevant relationship was present to the other load-independent conductance indices or the determined FT parameters (-Supplementary Tables).

\section{Cardiac Magnetic Resonance Imaging}

There was a negative correlation between the end-diastolic right ventricular volume (RV-EDVi: $110 \pm 22 \mathrm{~mL} / \mathrm{m}^{2}$ ) and the $\mathrm{RV}$ ejection fraction $(r=-0.53 ; p=0.033)$. RV ejection fraction was only moderately decreased (mean RV-EF: $41 \pm 8 \%$ ). CMR-derived cardiac index showed normal values (mean $\mathrm{CI}$ : $3.2 \pm 0.76 \mathrm{~L} / \mathrm{min} / \mathrm{m}^{2}$; - Table 2).

FT analysis revealed lower strain values for the right ventricle compared with the left ventricle in all three
Table 2 CMR data with feature tracking results

\begin{tabular}{|l|l|}
\hline CMR parameters & Results \\
\hline RV-EDVi $\left(\mathrm{ml} / \mathrm{m}^{2}\right)$ & $110 \pm 22(78-156)$ \\
\hline RV-ESVi $\left(\mathrm{ml} / \mathrm{m}^{2}\right)$ & $66 \pm 19(41-110)$ \\
\hline RV-SVi $\left(\mathrm{ml} / \mathrm{m}^{2}\right)$ & $44 \pm 9(32-67)$ \\
\hline RV-EF $(\%)$ & $41 \pm 8(25-52)$ \\
\hline $\mathrm{Cl}\left(\mathrm{L} / \mathrm{min} / \mathrm{m}^{2}\right)$ & $3.2 \pm 0.76(2.14-4.63)$ \\
\hline RV-GLS $(\%)$ & $-12.9 \pm 2.7$ \\
\hline RV-GRS $(\%)$ & $18.6 \pm 6.4$ \\
\hline RV-GCS (\%) & $-17.4 \pm 2.5$ \\
\hline LV-GLS (\%) & $-16.8 \pm 3.9$ \\
\hline LV-GRS (\%) & $20.9 \pm 4.6$ \\
\hline LV-GCS (\%) & $-22.9 \pm 3.2$ \\
\hline
\end{tabular}

Abbreviations: $\mathrm{Cl}$, cardiac index; $\mathrm{CMR}$, cardiac magnetic resonance; $E D V i$, end-diastolic volume index; EF, ejection fraction; ESVi, end-systolic volume index; RV, right ventricle; LV, left ventricle; SVi, stroke volume index.

Strain parameters: GCS, global circumferential strain; GLS, global longitudinal strain; GRS, global radial strain (always maximal global systolic strain).

Note: Data are means \pm standard deviation (range).

dimensions (GLS, GRS, and GCS; - Table 2). Statistically significant correlations were found for the following CMRderived parameters: RV-EF and RV-GCS $(r=-0.66, p=0.005)$, LV-EF and LV-GCS $(r=-0.53, p=0.034)$, LV-EF and LV-GLS $(r=-0.50, \quad p=0.048), \quad$ RV-GLS und LV-GCS $(r=0.66$, $p=0.006)$, LV-EF and RV-GLS $(r=-0.72, p=0.002)$, and LVGLS and LV-GCS $(r=0.56, p=0.024)$. For the following correlations, we found no statistically significant relationships: RV-EF and RV-GLS $(r=-0.41, p=0.114)$, RV-EF and RV-GRS $(r=-0.02, p=0.953)$, and LV-EF and LV-GRS $(r=0.49$, $p=0.057$ ). Additionally, we found no relevant relationship between strain parameters and QRS width or CPET parameters $\left(\mathrm{pVO}_{2}\right.$ and $\mathrm{O}_{2} / \mathrm{HR}$; - Supplementary Tables).

\section{Conductance}

On average, a significant increase in contractility was found under dobutamine infusion (-Fig. 2, patient example under -Supplementary Figure). This was demonstrated by the load-dependent systolic function parameter dp/dtmax (increase by $189 \%, p \leq 0.0001$ ), as well as by the load-independent systolic function parameters end-systolic elastance (Ees), preload recruitable stroke work (PRSW), and Starling's contractility index (SCI; increase by $167-229 \%, p \leq 0.0001$ ). Ees was overall reduced and showed significant increase under dobutamine (from $0.80 \pm 0.44$ to $1.89 \pm 0.72 \mathrm{~mm}$ $\mathrm{Hg} / \mathrm{mL}, \quad p \leq 0.001)$, Ea increased moderately from $1.00 \pm 0.26$ to $1.38 \pm 0.36 \mathrm{~mm} \mathrm{Hg} / \mathrm{mL}(p \leq 0.001)$, and, in consequence, the ratio Ea/Ees (ventricular-arterial coupling) showed a significant decrease (29\%) under dobutamine infusion (from $1.55 \pm 0.70$ to $0.84 \pm 0.39, p \leq 0.021$ ). The load-independent parameter of diastolic function end-diastolic elastance (Eed) remained nearly unchanged (from $0.11 \pm 0.70$ to $0.13 \pm 0.15 \mathrm{~mm} \mathrm{Hg} / \mathrm{mL}, \quad p=0,454)$ and 


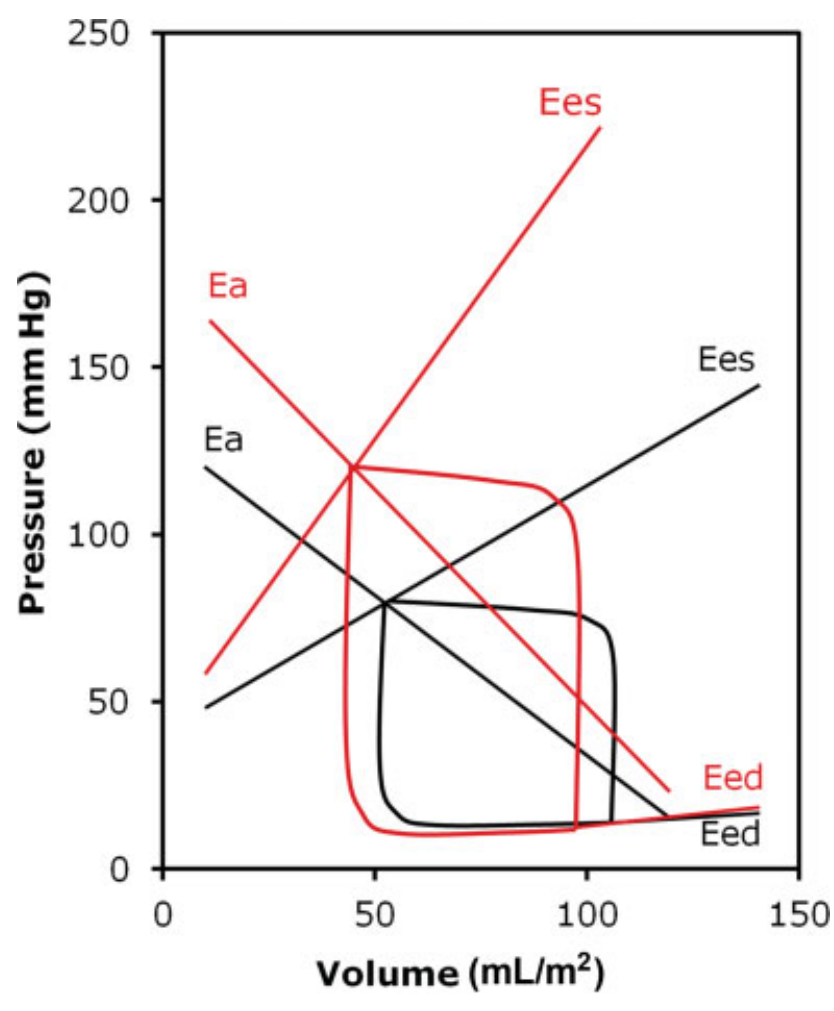

Fig. 2 Pressure-volume loops at baseline (black) and during dobutamine infusion (red) constructed from the mean values of the endsystolic and end-diastolic volume indices (ESVi and EDVi) and the endsystolic and end-diastolic pressures (ESP and EDP) for the entire patient group. The end-systolic and end-diastolic pressure-volume relationships (Ees and Eed) as well as the arterial elastance (Ea) are shown.

indicates no relevant change in myocardial stiffness under dobutamine stress. Diastolic relaxation and filling times (tau, tPFR, DFT) could be significantly reduced, whereas the peak filling time (PFT) remained constant (- Table $\mathbf{3}$ ).

We found an increase in cardiac index (100\%, $p \leq 0.0001)$, which, however, is not related to an increasing SV (not significant [n.s.]), but mainly caused by a rising heart rate ( $p \leq 0.0001$; - Table 3 ).

The dyssynchrony was higher in diastole (mean $30 \pm 4 \%$ baseline and $24 \pm 6 \%$ dobutamine) than in systole (mean $17 \pm 6 \%$ baseline and $19 \pm 7 \%$ dobutamine). Apical segments showed a higher dyssynchrony compared with basal segments. During dobutamine stress, dyssynchrony increased in systolic phase (n.s.) and decreased in diastolic phase $(p=0.001 ;$ - Table 3$)$.

\section{Discussion}

Interpretation of the Conductance Catheter Results

In the present study, cardiac index significantly increased under dobutamine infusion, whereas SV did not significantly increase. Higher cardiac index was therefore a result from a higher heart rate induced by $\beta$-adrenergic drug stimulation. The cardiopulmonary exercise data of our patients also suggested that the limited exercise capacity is mainly a result from an insufficient increase in SV and, to a lesser degree, from chronotropic incompetence. These results are in accordance with previously published stress studies ${ }^{8,9}$ that further demonstrated that in contrast to patients after atrial switch, patients with congenitally corrected transposition of the great arteries (ccTGA) could significantly increase their SV. ${ }^{10}$ This could suggest that the rigid atrial baffle in atrial switch might lead to a reduced atrial filling capacity and atrial function, which might also explain the absence of increase in peak filling rate (PFR). ${ }^{9}$ Increased tricuspid regurgitation under stress conditions could play an additional role. ${ }^{11}$ The negative correlation of the oxygen pulse $\left(\mathrm{O}_{2} / \mathrm{HR}\right)$ and Ea reflects the increased stroke work with increased vascular resistance.

In accordance with other studies, ${ }^{9,12}$ our patients showed a significant increase in contractility (Ees) under dobutamine stress (from $0.80 \pm 0.44$ to $1.89 \pm 0.72 \mathrm{~mm} \mathrm{Hg} / \mathrm{mL}, p$ $\leq 0.001$ ) reflecting adequate myocardial reserve. Additionally, ventricular stiffness (Eed) under dobutamine influence remained unchanged in our patients.

Ees adequately detects changes in systolic contractility ${ }^{13}$ and is used to quantify ventricular-arterial coupling (Ea/Ees) and thus the efficiency of ventricular work. Under stress, ventricular-arterial coupling showed a significant decrease in our patients (from $1.55 \pm 0.70$ to $0.84 \pm 0.39, p \leq 0.021$ ). Thus, ventricular stroke work under stress conditions became more effective in this patient group and this was primarily the result of rising contractility (Ees) under dobutamine infusion, whereas increase of Ea was only mild (from $1.00 \pm 0.26$ to $1.38 \pm 0.36 \mathrm{~mm} \mathrm{Hg} / \mathrm{mL}, p \leq 0.001$; - Table 3 and -Fig. 2). A systemic right ventricle is exposed to a chronic pressure load, and an additional volume load may appear in the presence of tricuspid insufficiency. The patients of the present study showed a mean Ees of $0.8 \mathrm{~mm} \mathrm{Hg} / \mathrm{mL}$, on average 27 years after atrial switch repair. A nearly identical Ees with an average of $0.9 \mathrm{~mm} \mathrm{Hg} / \mathrm{mL}$ was reported by Derrick et al in patients after Mustard repair with a comparable age. ${ }^{9}$ In the literature, normal right ventricles in subpulmonary position reach Ees values of 0.44 to $0.47 \mathrm{~mm} \mathrm{Hg} / \mathrm{mL}^{14,15}$ Ventricles with volume overload show lower contractility with an Ees of 0.24 to $0.35 \mathrm{~mm}$ $\mathrm{Hg} / \mathrm{mL}^{16}$ The investigations of Schlangen et al in patients with hypoplastic left heart syndrome (HLHS) demonstrated higher contractility of the right ventricle with an average Ees of 2.9 to $3.2 \mathrm{~mm} \mathrm{Hg} / \mathrm{mL}$ at rest and a significant increase up to 6.55 to $6.7 \mathrm{~mm} \mathrm{Hg} / \mathrm{mL}$ under load. ${ }^{17,18}$ This reflects good adaptation of the right ventricle to a chronic pressure load in this younger population (mean: 2.5 years) after Fontan's palliation. Healthy left ventricles in adults have Ees values of $\sim 2.0 \mathrm{~mm} \mathrm{Hg} / \mathrm{mL}$ with an age- and sex-specific increase. ${ }^{19,20}$ Ees can be measured up to $4.0 \mathrm{~mm} \mathrm{Hg} / \mathrm{mL}$ in cases of hypertrophied left ventricles, whereas, in contrast, cases of dilated insufficient left ventricle contractility are below an Ees of $1.0 \mathrm{~mm} \mathrm{Hg} / \mathrm{mL}^{19,21}$

In summary, Ees was within the range of previously published values for insufficient left ventricles. ${ }^{19,21}$ This finding reflects the discrepancy in our patient group: a subjective and objective (CPET) good exercise capacity stands in contrast with progressive right heart failure. 
Table 3 Conductance data (under baseline and dobutamine infusion)

\begin{tabular}{|c|c|c|c|c|c|c|c|c|}
\hline Hemodynamic parameters & \multicolumn{2}{|l|}{ Baseline } & \multicolumn{2}{|c|}{ Dobutamine } & \multicolumn{2}{|c|}{ Change (\%) } & \multicolumn{2}{|l|}{ Sign test } \\
\hline $\mathrm{HR}(/ \min )$ & \multicolumn{2}{|l|}{$53 \pm 9$} & \multicolumn{2}{|l|}{$99 \pm 25$} & \multicolumn{2}{|l|}{87} & \multicolumn{2}{|l|}{$\leq 0.0001$} \\
\hline $\mathrm{EDVi}\left(\mathrm{mL} / \mathrm{m}^{2}\right)$ & \multicolumn{2}{|l|}{$106 \pm 30$} & \multicolumn{2}{|l|}{$97 \pm 41$} & \multicolumn{2}{|l|}{-4} & \multicolumn{2}{|l|}{ n.s. } \\
\hline $\mathrm{ESVi}\left(\mathrm{mL} / \mathrm{m}^{2}\right)$ & \multicolumn{2}{|l|}{$53 \pm 25$} & \multicolumn{2}{|l|}{$44 \pm 37$} & \multicolumn{2}{|l|}{-10} & \multicolumn{2}{|l|}{ n.s. } \\
\hline $\mathrm{SVi}\left(\mathrm{mL} / \mathrm{m}^{2}\right)$ & \multicolumn{2}{|l|}{$46 \pm 9$} & \multicolumn{2}{|l|}{$50 \pm 10$} & \multicolumn{2}{|l|}{9} & \multicolumn{2}{|l|}{ n.s. } \\
\hline EF (\%) & \multicolumn{2}{|l|}{$48 \pm 12$} & \multicolumn{2}{|l|}{$59 \pm 20$} & \multicolumn{2}{|l|}{24} & \multicolumn{2}{|l|}{ n.s. } \\
\hline $\mathrm{Cl}\left(\mathrm{L} / \mathrm{min} / \mathrm{m}^{2}\right)$ & \multicolumn{2}{|c|}{$2.4 \pm 0.32$} & \multicolumn{2}{|c|}{$4.8 \pm 1.06$} & \multicolumn{2}{|l|}{100} & \multicolumn{2}{|l|}{$\leq 0.0001$} \\
\hline Pes $(\mathrm{mm} \mathrm{Hg})$ & \multicolumn{2}{|l|}{$84 \pm 14$} & $127 \pm 20$ & & 51 & & $\leq 0.0001$ & \\
\hline Ped $(\mathrm{mm} \mathrm{Hg})$ & $11.2 \pm 5$ & & $9.6 \pm 8.6$ & & -23 & & n. s. & \\
\hline $\mathrm{SW}(\mathrm{mm} \mathrm{Hg} \mathrm{mL)}$ & $6,868 \pm 2$ & & $11,947 \pm$ & 666 & 78 & & $\leq 0.0001$ & \\
\hline $\mathrm{dp} / \mathrm{dtmax}(\mathrm{mm} \mathrm{Hg} / \mathrm{s})$ & $657 \pm 12$ & & $1,848 \pm 2$ & & 189 & & $\leq 0.0001$ & \\
\hline $\mathrm{dp} / \mathrm{dtmin}(\mathrm{mm} \mathrm{Hg} / \mathrm{s})$ & $776 \pm 12$ & & $1,550 \pm$ & & 104 & & $\leq 0.0001$ & \\
\hline $\operatorname{PER}(\mathrm{mL} / \mathrm{s})$ & $601 \pm 29$ & & $914 \pm 31$ & & 67 & & $\leq 0.001$ & \\
\hline tPER (ms) & $201 \pm 51$ & & $133 \pm 49$ & & -32 & & $\leq 0.004$ & \\
\hline Tau (ms) & $40 \pm 10$ & & $26 \pm 6$ & & -35 & & $\leq 0.0001$ & \\
\hline PFR $(\mathrm{mL} / \mathrm{s})$ & $721 \pm 26$ & & $870 \pm 25$ & & 31 & & n.s. & \\
\hline tPFR (ms) & $595 \pm 61$ & & $413 \pm 43$ & & -30 & & $\leq 0.0001$ & \\
\hline DFT (ms) & $703 \pm 19$ & & $342 \pm 12$ & & -50 & & $\leq 0.0001$ & \\
\hline Ees $(\mathrm{mm} \mathrm{Hg} / \mathrm{ml})$ & $0.80 \pm 0$ & & $1.89 \pm 0$. & & 191 & & $\leq 0.001$ & \\
\hline PRSW (mm Hg) & $54 \pm 25$ & & $122 \pm 42$ & & 167 & & $\leq 0.001$ & \\
\hline $\mathrm{SCl}(\mathrm{mm} \mathrm{Hg} / \mathrm{mL} / \mathrm{s})$ & $2.46 \pm 2$ & & $10.1 \pm 6$. & & 229 & & $\leq 0.001$ & \\
\hline $\mathrm{Ea}(\mathrm{mm} \mathrm{Hg} / \mathrm{ml})$ & $1.00 \pm 0$ & & $1.38 \pm 0$ & & 41 & & $\leq 0.001$ & \\
\hline Ea/Ees & $1.55 \pm 0$. & & $0.84 \pm 0$ & & -29 & & $\leq 0.021$ & \\
\hline Eed $(\mathrm{mm} \mathrm{Hg} / \mathrm{ml})$ & $0.11 \pm 0$. & & $0.13 \pm 0$. & & 34 & & n.s. & \\
\hline Dyssynchrony & & & & & & & & \\
\hline Segments & Systole & Diastole & Systole & Diastole & Systole & Diastole & Systole & Diastole \\
\hline Apical 1 & $19 \pm 9$ & $36 \pm 6$ & $25 \pm 11$ & $33 \pm 10$ & 57 & -8 & n. s. & 0.035 \\
\hline Apical 2 & $22 \pm 11$ & $34 \pm 7$ & $23 \pm 12$ & $29 \pm 9$ & 21 & -12 & n. s. & 0.035 \\
\hline Mid & $17 \pm 7$ & $30 \pm 6$ & $15 \pm 17$ & $21 \pm 8$ & -7 & -27 & n. s. & 0.007 \\
\hline Base 2 & $12 \pm 7$ & $26 \pm 5$ & $13 \pm 9$ & $18 \pm 9$ & 39 & -32 & n. s. & 0.007 \\
\hline Base 1 & $17 \pm 8$ & $24 \pm 6$ & $16 \pm 10$ & $20 \pm 12$ & 17 & -18 & n. s. & n. s. \\
\hline Total & $17 \pm 6$ & $30 \pm 4$ & $19 \pm 7$ & $24 \pm 6$ & 11 & -20 & n. s. & 0.001 \\
\hline
\end{tabular}

Abbreviations: $\mathrm{Cl}$, cardiac index; DFT, diastolic filling time, dp/dtmax, maximum value of first derivative of pressure; $\mathrm{dp} / \mathrm{dtmin}$, minimum value of first derivative of pressure; Ea, arterial elastance, Ea/Ees, ventriculoarterial coupling; EDVi, end-diastolic volume index; Eed, end-diastolic stiffness; Ees, end-systolic elastance; EF, ejection fraction; ESVi, end-systolic volume index; HR, heart rate; n.s., not significant; Ped, end-diastolic pressure; tPER, time peak ejection rate; Pes, end-systolic pressure; tPFR, time peak filling rate; PRSW, preload recruitable stroke work; SCI, Starling's contractility index; SVi, stroke volume index; SW, stroke work; systole, from end-diastolic volume to end-systolic volume; diastole, from end-systolic volume to end-diastolic volume.

Note: Data are means \pm standard deviation.

\section{Conductance-Derived Dyssynchrony}

Inhomogeneous contraction that reinforces the existing problem of cardiac dysfunction could be another relevant problem. ${ }^{22}$ Therefore, the assessment of dyssynchrony could be helpful for a complete evaluation of cardiac function and possibly of crucial relevance for further treatment. ${ }^{23}$ Cardiac resynchronization therapy (CRT) has the potential to improve ventricular function in some cases. ${ }^{23,24}$ Pressurevolume loop analyses using conductance catheter technique provides sensitive detection of systolic and diastolic dyssynchrony with good temporal and spatial resolution. ${ }^{4,25}$ To the best of our knowledge, this is the first study that investigated dyssynchrony in atrial switch patients by conductance analysis. Due to the lack of values in humans, interpretation of our results is difficult. In a pig model, a physiological systolic left ventricular dyssynchrony due to intraventricular delay was shown to be less than $10 \%{ }^{26}$ Dyssynchrony values were clearly elevated in our investigations, more diastolic than 
systolic, and more pronounced apically. Similar findings were found in patients with coronary artery disease as well as in left-sided heart failure patients. ${ }^{4}$

Under dobutamine stress, dyssynchrony did not significantly increase in systole (17 vs. $19 \%$ ), but decreased significantly in diastole (30 vs. $24 \%$ ). A subdivision into early (Pes to PFR) and late diastole (PFR to Ped) showed that dyssynchrony decreased on average especially in late diastole ( -4 vs. $-31 \%$ ), but did not reach statistical significance. In contrast, Kurita et al showed that heart failure patients with nonischemic heart disease developed augmented systolic dyssynchrony under tachycardiac pacing (with a significant increase in systolic dyssynchrony from $37-44$ to $43-48 \%$ ). ${ }^{25}$ However, their study protocol and patient selection are not comparable to our setting. Patients were much older (mean: $62 \pm 11 / 62 \pm 14$ years for the heart failure group), and myocardial remodeling in the evaluated left ventricles was possibly advanced. Diastolic dyssynchrony was not evaluated in their patient group. We suspect that our patients (with a systemic right ventricle) may also develop an increasing systolic dyssynchrony in the long term. To prove this hypothesis, repeated conductance examinations at longer time intervals would be necessary.

\section{CMR-Derived Data for Functional Assessment}

CMR FT could have the potential to provide noninvasive parameters to assess the beginning of deterioration of the RV function. Systolic strain values for the right ventricle were distinctly lower than in a normal left ventricle, whereas ejection fraction was only moderately decreased. Preexisting data from the literature suggest that the normal left ventricle global longitudinal systolic strain (LV-GLS) has a cutoff value between -17.3 and $-21.5 \%{ }^{27}$ For the systemic RV in our patients, we found markedly lower GLS values of $-12.9 \pm 2.7 \%$. Our evaluated strain values were comparable to recently published reference values by Tutarel et al. ${ }^{7}$ However, a major methodical difference was that in the present study radial strain was assessed in a four-chamber view and not in the short axis. The global systolic strain parameters revealed lower values for the systemic right ventricle than in the subpulmonary left ventricle (in all three dimensions). Global longitudinal systolic strain was decreased for the right ventricle after atrial switch, whereas global circumferential systolic strain showed higher values. This relationship, normally to be expected from a left ventricle, reflects functional adaptation mechanisms of the right ventricle to chronic pressure load. ${ }^{28}$ Salehi Ravesh et al recently presented FT data from younger patients with HLHS. In their study population with a median age of 4.9 years, GLS of the systemic right ventricle had distinctly higher values than in our older population (mean age: 28.2 years). ${ }^{29}$ In concordance with Tutarel et al, ${ }^{7}$ we found a good correlation between RV-EF and RV-GCS in our CMR study as well as between LV-EF and LV-GCS. Another interesting finding was an interventricular relationship of the strain parameters (RV-GLS and LV-GCS), showing that these contractility parameters may have an association in cases of decline. In this context, the correlation of LV-EF and RV-GLS in our data supports this hypothesis.

\section{Interrelationship between Parameters of Systemic RV Function}

All patients were grouped in NYHA classes I to II, which is consistent with many other comparable studies. ${ }^{1}$ There was no statistically significant correlation between the loadindependent contractility indices at rest and the global systolic strain parameters-thinking about replacing the invasive method by a noninvasive technique. One possible reason for our observation might be that data acquisition for the conductance indices is performed at different sites of the right systemic ventricle than in the FT analysis. Whereas strain evaluations were referred to the four-chamber view and the mid short axis, the conductance data were based (ideally) on the whole right ventricle. Thus, a comprehensive analysis in this patient group remains difficult, because the different invasive and noninvasive examinations as performed in our study did not show a clear interrelation. Helsen et al recently reported comparable findings in terms of the relationship of exercise testing parameters with functional echocardiographic or CMR-derived parameters for a systemic RV. ${ }^{30}$ We additionally could not derive any relevant relation between the invasive measured indices under dobutamine stress and the exercise capacity parameters in our patients.

\section{Limitations}

Patients with severe heart failure were excluded from this study. FT data were only evaluated by one investigator (no interobserver variability available). This is a single-center cross-sectional study and the number of patients with d-TGA after atrial switch operation is limited. Follow-up studies are not possible anymore because arterial switch correction is now the treatment of choice. Conductance data for the systemic right ventricle are limited; therefore, interpretation is difficult and their prognostic relevance has to be evaluated in other cohorts, for example, patients with HLHS. Optimally, the conductance catheter is placed in a straight position from the aortic valve to the ventricular apex. The distance from the pigtail to the first measurement electrode is $\sim 2 \mathrm{~cm}$. Irritation of the walls must be avoided as it might trigger cardiac arrhythmia, especially under dobutamine stress. This may require a slight pullback of the catheter from the apical position. Therefore, if the apical region is highly dyssynchronous, this methodology might underestimate dyssynchrony. Due to the arterial approach of the conductance catheter, the inflow tract may not be sufficiently represented in the conductance data.

\section{Conclusion}

Our conductance analysis demonstrates that systemic RV elastance (Ees) in the long term corresponds to values reported for an insufficient left ventricle. Increased cardiac output under stress (dobutamine infusion) was mainly achieved via an increasing heart rate and not by a rise in SV.

Another interesting finding was that there was no statistically significant correlation between the load- 
independent contractility indices and the global systolic strain parameters. Nevertheless, CMR-based strain analysis using FT technique may provide a helpful noninvasive diagnostic tool for early detection of deterioration.

Longitudinal studies with lager cohorts using either conductance technology or CMR (as well as CPET and clinical endpoints) are necessary to investigate subtle changes in systemic RV function.

In all patients, conductance analysis revealed significant dyssynchrony, which was more present in diastole than in systole. To the best of our knowledge, this is the first study presenting conductance-derived mechanical dyssynchrony in patients after atrial switch. Whether this is an independent process or precursor of incipient contractility loss, could be a subject for further studies.

\section{Equal Authorships}

Both Andreas Hornung and Daniela Vollmer were involved in data collection and interpretation. Furthermore, D.V. was responsible for the study design and A.H. for writing of the manuscript.

\section{Funding}

This study was financially supported by the Fördergemeinschaft Deutsche Kinderherzzentren, Bonn, Germany.

\section{Conflict of Interest}

On behalf of all authors, the corresponding author states that there is no conflict of interest.

\section{Acknowledgments}

We thank the "Fördergemeinschaft Deutsche Kinderherzzentren" (Bonn, Germany) and the "Stiftung zur Förderung der Erforschung von Zivilisationserkrankungen” (Baden-Baden, Germany) for financial support. We also thank our colleague Dr. Winfried Baden for evaluation of the CPET studies and our colleagues from the Department of Radiology for the good cooperation.

\section{References}

1 Dos L, Teruel L, Ferreira IJ, et al. Late outcome of Senning and Mustard procedures for correction of transposition of the great arteries. Heart 2005;91(05):652-656

2 Apitz C, Latus $\mathrm{H}$, Binder W, et al. Impact of restrictive physiology on intrinsic diastolic right ventricular function and lusitropy in children and adolescents after repair of tetralogy of Fallot. Heart 2010;96(22):1837-1841

3 Baan J, van der Velde ET, de Bruin HG, et al. Continuous measurement of left ventricular volume in animals and humans by conductance catheter. Circulation 1984;70(05):812-823

4 Steendijk P, Tulner SA, Schreuder JJ, et al. Quantification of left ventricular mechanical dyssynchrony by conductance catheter in heart failure patients. Am J Physiol Heart Circ Physiol 2004;286 (02):H723-H730

5 Dubowy KO, Baden W, Bernitzki S, Peters B. A practical and transferable new protocol for treadmill testing of children and adults. Cardiol Young 2008;18(06):615-623

6 Hor KN, Baumann R, Pedrizzetti G, et al. Magnetic resonance derived myocardial strain assessment using feature tracking. J Vis Exp 2011;(48):2356
7 Tutarel O, Orwat S, Radke RM, et al. Assessment of myocardial function using MRI-based feature tracking in adults after atrial repair of transposition of the great arteries: reference values and clinical utility. Int J Cardiol 2016;220:246-250

8 Paul MH, Wessel HU. Exercise studies in patients with transposition of the great arteries after atrial repair operations (Mustard/Senning): a review. Pediatr Cardiol 1999;20(01): 49-55, discussion 56

9 Derrick GP, Narang I, White PA, et al. Failure of stroke volume augmentation during exercise and dobutamine stress is unrelated to load-independent indexes of right ventricular performance after the Mustard operation. Circulation 2000;102(19, Suppl 3): III154-III159

10 Fratz S, Hager A, Busch R, et al. Patients after atrial switch operation for transposition of the great arteries can not increase stroke volume under dobutamine stress as opposed to patients with congenitally corrected transposition. Circ J 2008;72(07): 1130-1135

11 Budts W, Scheurwegs C, Stevens A, Moons P, Van Deyk K, Vanhees L. The future of adult patients after Mustard or Senning repair for transposition of the great arteries. Int J Cardiol 2006;113(02): 209-214

12 Vogel M, Derrick G, White PA, et al. Systemic ventricular function in patients with transposition of the great arteries after atrial repair: a tissue Doppler and conductance catheter study. J Am Coll Cardiol 2004;43(01):100-106

13 Rahko PS. Comparative efficacy of three indexes of left ventricular performance derived from pressure-volume loops in heart failure induced by tachypacing. J Am Coll Cardiol 1994;23(01):209-218

14 Sanz J, García-Alvarez A, Fernández-Friera L, et al. Right ventriculo-arterial coupling in pulmonary hypertension: a magnetic resonance study. Heart 2012;98(03):238-243

15 McCabe C, White PA, Hoole SP, et al. Right ventricular dysfunction in chronic thromboembolic obstruction of the pulmonary artery: a pressure-volume study using the conductance catheter. J Appl Physiol (1985) 2014;116(04):355-363

16 Latus H, Binder W, Kerst G, Hofbeck M, Sieverding L, Apitz C. Right ventricular-pulmonary arterial coupling in patients after repair of tetralogy of Fallot. J Thorac Cardiovasc Surg 2013;146(06): 1366-1372

17 Schlangen J, Fischer G, Petko C, et al. Arterial elastance and its impact on intrinsic right ventricular function in palliated hypoplastic left heart syndrome. Int J Cardiol 2013;168(06):5385-5389

18 Logoteta J, Ruppel C, Hansen JH, et al. Ventricular function and ventriculo-arterial coupling after palliation of hypoplastic left heart syndrome: a comparative study with Fontan patients with LV morphology. Int J Cardiol 2017;227:691-697

19 Chen CH, Fetics B, Nevo E, et al. Noninvasive single-beat determination of left ventricular end-systolic elastance in humans. J Am Coll Cardiol 2001;38(07):2028-2034

20 Redfield MM, Jacobsen SJ, Borlaug BA, Rodeheffer RJ, Kass DA. Ageand gender-related ventricular-vascular stiffening: a communitybased study. Circulation 2005;112(15):2254-2262

21 Ky B, French B, May Khan A, et al. Ventricular-arterial coupling, remodeling, and prognosis in chronic heart failure. J Am Coll Cardiol 2013;62(13):1165-1172

22 Chow PC, Liang XC, Lam WW, Cheung EW, Wong KT, Cheung YF. Mechanical right ventricular dyssynchrony in patients after atrial switch operation for transposition of the great arteries. Am J Cardiol 2008;101(06):874-881

23 Janousek J, Tomek V, Chaloupecký VA, et al. Cardiac resynchronization therapy: a novel adjunct to the treatment and prevention of systemic right ventricular failure. J Am Coll Cardiol 2004;44 (09):1927-1931

24 Cecchin F, Frangini PA, Brown DW, et al. Cardiac resynchronization therapy (and multisite pacing) in pediatrics and congenital heart disease: five years experience in a single institution. J Cardiovasc Electrophysiol 2009;20(01):58-65 
25 Kurita T, Onishi K, Dohi K, et al. Impact of heart rate on mechanical dyssynchrony and left ventricular contractility in patients with heart failure and normal QRS duration. Eur J Heart Fail 2007;9 (6-7):637-643

26 A'roch R, Steendijk P, Oldner A, et al. Left ventricular mechanical dyssynchrony is load independent at rest and during endotoxaemia in a porcine model. Acta Physiol (Oxf) 2009;196(04): 375-383

27 Claus P, Omar AMS, Pedrizzetti G, Sengupta PP, Nagel E. Tissue tracking technology for assessing cardiac mechanics: principles, normal values, and clinical applications. JACC Cardiovasc Imaging 2015;8(12):1444-1460
28 Pettersen E, Helle-Valle T, Edvardsen T, et al. Contraction pattern of the systemic right ventricle shift from longitudinal to circumferential shortening and absent global ventricular torsion. J Am Coll Cardiol 2007;49(25):2450-2456

29 Salehi Ravesh M, Rickers C, Bannert FJ, et al. Longitudinal deformation of the right ventricle in hypoplastic left heart syndrome: a comparative study of 2D-feature tracking magnetic resonance imaging and 2D-speckle tracking echocardiography. Pediatr Cardiol 2018;39(06):1265-1275

30 Helsen F, De Meester P, Van De Bruaene A, et al. Right ventricular systolic dysfunction at rest is not related to decreased exercise capacity in patients with a systemic right ventricle. Int J Cardiol 2018;260:66-71 